\title{
COGNITIVE RADIO RESOURCE ALLOCATION BASED ON THE IMPROVED QUANTUM GENETIC ALGORITHM
}

\author{
Bin Han, ${ }^{*}$ Hong Jiang, ${ }^{*}$ Ying Luo, ${ }^{*}$ and Jinzhi Zhou*
}

\begin{abstract}
In the spectrum allocation of cognitive radio (CR) network, the problems of local optimum and premature convergence remain challenging. To further improve the efficiency of the spectrum allocation, this paper proposes a novel method based on an improved quantum genetic algorithm. This is an algorithm that is designed to dynamically adjust the quantum rotation angle to speed up the convergence rate. In particular, the variation threshold was introduced to the mutation operation on chromosomes, establishing new interference constraint rules in the process of spectrum allocation. The simulation work was implemented for validation, and the results revealed that the proposed methodology achieved better average benefits of the CR network for a reasonable allocation of the spectrum.
\end{abstract}

\section{Key Words}

Cognitive radio, quantum genetic algorithm, spectrum allocation, variation threshold, continuous function

\section{Introduction}

In recent year, wireless network technologies have been widely applied in the fields of industrial automation [1], smart power grid [2], robots control [3], unmanned aerial vehicle (UAV) infrastructure [4], etc. [5]. Although they are characterized by impressive cost-effective energy consumption, high flexibility, and strong expansibility, the increased demand for wireless communication brought out new problems, where the spectrum resource scarcity is highlighted [6]. Mitola [7] creatively proposed the concept of cognitive radio (CR) on the basis of software radio to effectively solve the problems of scarce spectrum resource and low spectrum. CR is powerful to detect the spectrum state in real time without affecting the primary users. Particularly, it is quite helpful in redistributing the idle

* School of Information Engineering, Southwest University of Science and Technology, Mianyang, People's Republic of China; e-mail: \{hanbin, jianghong, luoying, zhoujinzhi\}@swust.edu.cn Corresponding author: Bin Han

Recommended by Prof. Anmin Zhu

(DOI: 10.2316/J.2019.206-0233) spectrum as needed by cognitive users to hit a higher effective rate. At present, the dynamic spectrum allocation technology [8] in this regard has become one of the research highlights as it works well in solving the problem of spectrum scarcity in a way of better utilizing the spectrum resource.

The constant transformation of spectrum information makes it necessary to take care of the efficiency of allocation algorithm. The current algorithms mainly involve graph colouring, game theory, and auction bidding. Among them, the graph-colouring theory proposed by Driouch et al. [9] has been widely used for the spectrum allocation, but still it is facing some problems such as uneven distribution and massive time overhead. Xiao et al. [10] proposed an interference alignment algorithm based on the game theory, which may eliminate the interference between primary users and secondary users and the interference among secondary users. Though this approach effectively improves the transmission rate, it is likely to be trapped in the local optimum. Sengupta et al. [11] proposed a dynamic spectrum allocation algorithm based on a bidding model, which availably increased the probability of successful bidding for cognitive users, but this merit is offset by its lower distribution efficiency and larger time overhead. The intelligent optimization algorithm, as a current research highlight, has been widely used in the spectrum allocation of CR [12] [13]. A spectrum allocation algorithm based on the genetic particle swarm is proposed [14] as it prevents the algorithm from being premature by introducing the particle swarm reconstruction mutation operator with better performance in terms of network and convergence. Nonetheless, being trapped in the local optimum is likely to occur. In [15], a unique spectrum allocation algorithm based on quantum genetic algorithm (QGA) is proposed. This algorithm is doing better to obtain optimal solution and convergence speed than the genetic algorithm (GA), which cannot avoid falling into local optimization in the iterative process. In [16], another concept is derived from a QGA based on simulated annealing. As an operator, the combination of simulated annealing algorithm and QGA may overcome the precocity convergence problem easily produced thereby and benefit from the two algorithms complementing each other. In [17], the CR spectrum was 
allocated with the help of the improved QGA (IQGA). By establishing an interference constraint matrix, the fairness between cognitive users and the efficiency of the network system were effectively enhanced.

To tackle the problem of slow convergence speed and local optimum, this paper proposes a new kind of algorithm that has incorporated the IQGA based on the colouring spectrum allocation model of graph theory. The algorithm not only effectively solves the problems that may be found in the conventional QGAs, but also it improves the average benefits of the network to achieve a reasonable allocation of spectrum.

The rest of this paper is organized as follows: related work is introduced in Section 1, and the spectrum allocation model is presented in Section 2. The spectrum allocation from the IQGA is described in Section 3. In Section 4, IQGA convergence analysis is given. Modelling results are discussed in Section 5 and this work is summarized in Section 6.

\section{Spectrum Allocation Model}

Using the graph-colouring model, the spectrum allocation problem [18] in this paper is presented by different matrices, including spectrum matrix $A$, utility matrix $B$, interference constraint matrix $C$, and interference-free allocation matrix $F$. The number of cognitive users of the network system in the paper is set as $N$, and that of authorized users and channels as $M$. In practical application, the time for cognitive wireless network to carry out spectrum allocation may be very short compared with the time of spectrum environment change. It is assumed that users' geographic location and available spectrum resources are static, that is matrices $A, B$, and $C$ remain unchanged within one distribution cycle [15]. The specific definitions are as follows:

1. Available spectrum matrix $A$

$$
A=\left\{a_{n, m} \in\{0,1\}\right\}_{N \times M}
$$

where $a_{n, m}=1$ indicates that cognitive user $n$ is allowed to use channel $m$; and $a_{n, m}=0$ means that the use of channel $m$ is not allowed.

2. Interference constraint matrix $C$

$$
C=\left\{c_{n, k, m} \mid c_{n, k, m} \in\{0,1\}\right\}_{N \times N \times M}
$$

It is a three-dimensional matrix. When channel $m$ is being used by cognitive users $n$ and $k$ at the same time, and the channel is interfered, then $c_{n, k, m}=1$; if there is no interference in this case, then $c_{n, k, m}=0$. When $n=k$ and $c_{n, k, m}=1-a_{n, m}$, then the interference constraint matrix $C$ is only determined by the available spectrum matrix $A$.

3. Utility matrix $B$

$$
\begin{gathered}
B=\left\{b_{n, m} \mid b_{n, m}>0\right\}_{N \times M} \\
b_{n, m}=W_{m} \log _{2}\left(1+\frac{p_{n, m} h_{n, m}}{n_{0}}\right) \geq b_{n, m}^{t h}
\end{gathered}
$$

where $B$ represents the throughput capacity obtained when cognitive user $n$ uses channel $m$. When $b_{n, m}=\alpha$, it indicates the gain obtained by cognitive user $n$ using the channel $m$ is $\alpha$; when $b_{n, m}=0$, it indicates that cognitive user $n$ is not allowed to use channel $m$. Where $W_{m}$ is the bandwidth of channel $m, p_{n, m}$ is the power used for transmission when user $n$ occupies channel $m$, so the power matrix is represented by $P, P=\left\{p_{n, m} \mid p_{n, m} \in\left[0, p_{n}^{t h}\right]\right\}$, and $\sum_{m=1}^{M} p_{n, m} \leq p_{n}^{\max }$, $h_{n, m}$ is the fading of the channel when user $n$ transmits through channel $m, h_{n, m}=d_{n, t}^{-\gamma}\left|h_{n, t}\right|$, where $d_{n, t}$ represents the physical distance between user $n$ and terminal node $t . \gamma$ shows the path fading coefficient, $h_{n, t}$ is the Rayleigh fading and represented by the zero-mean Gaussian model, that is $h_{n, t} \sim \mathrm{CN}(0,1)$. $b_{n, m}^{t h}$ denotes the minimum transmission rate required for user $n$.

4. Interference-free distribution matrix $F$

$$
F=\left\{f_{n, m} \mid f_{n, m} \in\{0,1\}, f_{n, m} \leq a_{n, m}\right\}_{N \times M}
$$

When $f_{n, m}=1$, it indicates that channel $m$ is assigned to user $n$; otherwise, $f_{n, m}=0$. The interference-free allocation matrix $F$ is required to satisfy the interference-free restriction condition:

$$
\begin{aligned}
& f_{n, m}+f_{k, m} \leq 1, \text { if } c_{n, k, m}=1, \\
& \quad \forall 0<n, \quad k<N, \quad 0<m<M
\end{aligned}
$$

By defining an interference-free allocation matrix, the gain obtained by a single cognitive user $n$ is defined as $r_{n}=\sum_{m=1}^{M} f_{n, m} \cdot b_{n, m}$, aiming to guarantee the transmission quality of users. Let $r_{n} \geq r_{n}^{t h}$, then the gains of all cognitive users form a matrix $R=\left\{r_{n}=\right.$ $\left.\sum_{m=1}^{M} f_{n, m} \cdot b_{n, m}\right\}_{N \times 1}$. If all interference-free spectrum allocation sets are defined as $\Lambda(A, C)_{N \times M}$ and the paper may use the average network efficiency $U(R)$ as an objective function, it can be expressed by the following formula:

$$
U(R)=\frac{1}{N} \sum_{n=1}^{N} \sum_{m=1}^{M} f_{n, m} \cdot b_{n, m}
$$

At the same time, the time overhead indicator was also introduced to evaluate the spectrum allocation performance to further verify the performance of the proposed algorithm. By defining $t$ as the time cost for each cycle, the total time overhead of the algorithm was equal to the number of cycles multiplied by $t$. The number of cycles was equal to the matrix norm $\|A\|_{m 1}$ of the matrix $F$. Here $T$ is the total time overhead of the algorithm.

$$
\left\{\begin{array}{l}
\text { LOOP }=\|F\|_{m 1}=\sum_{n=1}^{N} \sum_{m=1}^{M} f_{n, m} \\
T=t \times \mathrm{LOOP}
\end{array}\right.
$$

Therefore, the spectrum allocation problem in this work was described as the following optimization problem:

$$
\begin{aligned}
& \left(F^{*}, P^{*}\right)=\underset{F \in \Lambda(A, C)}{\arg \max } U(R) \\
& T^{*}=\min T \\
& \operatorname{s.t}\left\{\begin{array}{l}
A=\left\{a_{n, m} \in\{0,1\}\right\}_{N \times M} \\
C=\left\{c_{n, k, m} \mid c_{n, k, m} \in\{0,1\}\right\}_{N \times N \times M} \\
F=\left\{f_{n, m} \mid f_{n, m} \in\{0,1\}, f_{n, m} \leq a_{n, m}\right\}_{N \times M} \\
\sum_{m=1}^{M} p_{n, m} \leq p_{n}^{\max }, r_{n} \geq r_{n}^{t h}
\end{array}\right.
\end{aligned}
$$




\section{Spectrum Allocation of the Improved Quantum Genetic Algorithm}

Currently, the QGA works better than the classical GA [19] in terms of the distribution effect on the spectrum allocation, yet still facing problems in terms of premature convergence and being trapped in the local optimum [20]. Therefore, it is an urgent need to explore some sort of global optimization algorithm with more ergodic properties. Improvements from the following aspects are mainly focused in the paper. First, the quantum rotation angle is adjusted dynamically to speed up the convergence of the algorithm. Then, the variation threshold is set to measure the chromosomal variation, making it possible that new interference constraint rules are established in the spectrum allocation process.

\subsection{Chromosome Initialization}

For most of the conventional QGAs, a fixed coding system may be taken to initialize the chromosomes, leaving the population obtained to be too single. For this reason, this work introduced the chaos optimization algorithm for the population initialization, which shows better property than the random search and is more capable of finding out the locally optimal solution [18].

\subsection{Chromosomal Measurement}

The chromosomes of the population $Q(g)$ were measured to obtain a set of states $V(g)=\left\{v_{1}^{g}, v_{2}^{g}, \ldots, v_{s}^{g}\right\}$. The qubit of each chromosome was changed from the superposition states of $\mid 0>$ and $\mid 1>$ to the binary solutions corresponding to the objective function. The value of $j$ in $v_{i}^{g}$ was determined by $\alpha_{i j}^{g}(j=1,2, \ldots, l)$ of $q_{i}^{g}$, with the specific formula as follows [21]:

$$
v_{i j}^{g}= \begin{cases}0, & \left|\alpha_{i j}^{g}\right|^{2}>\operatorname{rand}(0,1) \\ 1, & \left|\alpha_{i j}^{g}\right|^{2} \leq \operatorname{rand}(0,1)\end{cases}
$$

\subsection{Dynamic Adjustment of the Quantum Rotation Angle}

In the QGA, the quantum rotation gate operation was used to update the gene qubit of quantum chromosomes. The purpose of the rotation gate was to obtain the optimal solution by increasing the probability that each qubit on the chromosome may be converged to 0 or 1 . The quantum rotation gate is expressed as

$$
\left\{\begin{array}{l}
G(\theta)=\left[\begin{array}{ll}
\cos (\theta) & -\sin (\theta) \\
\sin (\theta) & \cos (\theta)
\end{array}\right] \\
\theta=\Delta \theta \cdot S
\end{array}\right.
$$

where $\mathrm{G}(\theta)$ is the quantum rotation gate, $\theta$ is the quantum rotation angle, $\Delta \theta$ is the size of the rotation angle, and $S$ is the direction of the angle rotation.
If the $j$ th quantum chromosome gene in chromosome $i$ is $\left[\alpha_{i j}, \beta_{i j}\right]^{T}$, and it mutates to $\left[\alpha_{i j}^{\prime} \beta_{i j}^{\prime}\right]^{T}$ after the quantum rotation gate operation, then the update process is given as

$$
\left[\begin{array}{cc}
\alpha_{i j}^{\prime}, & \beta_{i j}^{\prime}
\end{array}\right]^{T}=G\left(\theta_{i j}\right)\left[\alpha_{i j}, \quad \beta_{i j}\right]^{T}
$$

The chromosome renewal for the conventional QGA was achieved through the quantum rotation angle $\theta$ with the determined size and direction. The algorithm is likely to be trapped in the local optimum when the selected amplitude is too large, whereas the convergence speed slows down when the selected amplitude is too small. Therefore, under a variable step search strategy, the size of the rotation angle was measured by obtaining the Hamming distance. The distance determined made it possible for the rotation angle to be adjusted in an adaptive manner [22]. The specific formula of the rotation angle is as follows:

$$
\left\{\begin{array}{l}
\theta=\theta_{\min }+k\left(\theta_{\max }-\theta_{\min }\right) \\
k=H(D, Z) / l
\end{array}\right.
$$

where $D$ is the binary solution of the individual to be evolved, $Z$ is the binary solution of the best individual, $l$ is the length of the chromosome, $k$ is the coefficient of adjustment of rotation angle $\theta, H(D, Z)$ represents the Hamming distance between individuals $D$ and $Z$, and $\theta_{\text {min }}$ is the shortest quantum rotation angle valued as $0.005 \pi$, and $\theta_{\max }$ is the maximum quantum rotation angle valued as $0.1 \pi$ [23].

From (13), it is concluded that the current individual has a lower fitness degree, and the corresponding quantum rotation angle is smaller when there is a shorter Hamming distance between the current and the optimal individuals. Consequently, the search range was reduced to get the local search, which improved the algorithm search accuracy and convergence speed. Furthermore, the search scope was expanded to achieve the universal search, which ultimately speeded up the search process of the algorithm.

\subsection{Variation Threshold Setting}

Regarding the conventional QGA, individuals are selected with a certain probability with a certain degree of blindness. The variation threshold is then set to determine whether the current chromosome needs to be mutated in this work, so as to effectively avoid the blindness of the conventional mutation operation in the process of individual mutation [21]. The variation threshold conditions were set as follows: (1) the Hamming distance between the binary solution $v_{i}$ of the current chromosome and the binary solution $v_{b}$ of the optimal chromosome was shorter than $0.5 l ;(2)\left(f_{b}-f_{i}\right) / f_{b} \leq 0.1$, where $f_{i}$ and $f_{b}$ were the fitness values of the current chromosome $i$ and the optimal chromosome, respectively. The current chromosome $i$ was subjected to the mutation processing when the two above-mentioned conditions were satisfied. 


\subsection{New Interference Constraint Rules}

The ownership of channel $m$ was determined according to the following formula when cognitive users $n$ and $k$ used channel $m$ simultaneously, and $c_{n, k, m}=1$ in the spectrum allocation process [24], [25]:

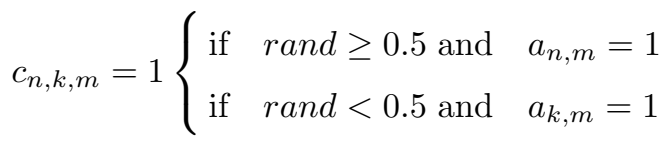

where rand is the random number between $[0,1], a_{n, m}=1$ means that cognitive user $n$ may use channel $m, a_{k, m}=1$ means that cognitive user $k$ may use channel $m$, and $c_{n, k, m}=1$ means that cognitive users $n$ and $k$ may not use channel $m$ simultaneously. From (14), when two cognitive users competed for the same channel, the algorithm determined the ownership of the channel by the random number rand. The interference constraint achieved through the above equation came along with some blindness, being unfavourable to the improvement of network efficiency and the fairness among cognitive users.

For this reason, a new interference constraint rule was designed in this paper: when the cognitive users $n$ and $k$ were interfered at the channel $m$ and the system did not know how to allocate channel, the ownership of the channel may be determined by calculating the degree of the demand for the channel $m$ of the two users. In this work, the degree of the user's demand for the channel was jointly determined from two aspects. The first one was the revenue of user from the channel, that is the throughput capacity utility. The second one was the number of channels already occupied by user. The purpose was to ensure the equalization of channel allocation.

As mentioned above, let us first use $u$ to represent the cognitive user who wanted to compete the ownership of channel $m$, where $u$ belonged to the set $\Phi$. Then, from (4), we used the throughput capacity $b_{u, m}$ to denote the revenue of user $u$. Finally, it is assumed that the number of channels occupied by the user $u$ was $s_{u}$. Therefore, the current degree of the user's demand for the channel $m$ $\left(X_{u, m}\right)$ can be expressed as

$$
X_{u, m}=w_{b, u} \frac{b_{u, m}}{\sum_{u \in \Phi} b_{u, m}}+w_{s, u} \frac{s_{u}}{N}
$$

where $w_{b, u}, w_{s, u}$ represent the weight of throughput capacity and channel occupancy, respectively. The definition of weight dynamically determined the degree of preference between capacity and channel occupancy.

Consequently, the ownership of channel $m$ was determined by comparing two users' joint utility $X$. Let us take the two cognitive users $n$ and $k$ as an example:

$$
\begin{cases}a_{n, m}=1, a_{k, m}=0, & \text { if } a_{n, m} \geq a_{k, m} \\ a_{n, m}=0, a_{k, m}=1, & \text { otherwise }\end{cases}
$$

\subsection{Proposed Algorithm}

Using $U(R)$ as the fitness function, the steps to improve the spectrum allocation algorithm of QGA are as follows:

1. Set the spectrum matrix $A$, the interference constraint matrix $C$, and the efficiency matrix $B$, where $A=\left\{a_{n, m} \in\{0,1\}\right\}_{N \times M}, C=\left\{c_{n, k, m} \mid c_{n, k, m} \in\right.$ $\{0,1\}\}_{N \times N \times M}, B=\left\{b_{n, m} \mid b_{n, m}>0\right\}_{N \times M}$, and then calculate the length of the chromosome $l=$ $\sum_{n=1}^{N} \sum_{m=1}^{M} l_{n, m}$. This was followed by recording the subscripts $n$ and $m$ under the element 1 in $A$ and saving them in $A_{1}=\left\{(n, m) \mid a_{n, m}=1\right\}$ in a gradually increasing manner;

2. Let $g=0$, initialize the encoding of chromosome $Q(g)=\left\{q_{1}^{g}, q_{2}^{g}, \ldots, q_{s}^{g}\right\}$

3. Measure $Q(g)$ to obtain the measured value $V(g)=$ $\left\{v_{1}^{g}, v_{2}^{g}, \ldots, v_{s}^{g}\right\}$

4. Map the $j$ th bit of the measured value $v_{i}^{g}(i=1,2, \ldots, s)$ to $f_{n, m}$, where $(n, m)$ is the $j$ th element $(j=1,2, \ldots, l)$ of $L_{1}$, and for all $m(0 \leq m \leq M)$, look for all cognitive users $n$ and $k$ satisfying $c_{n, k, m}=1$ in matrix $C$, and check whether the two measured values $F(n, m)$ and $F(k, m)$ in matrix $F$ are both 1 ; if they were both 1 , the new interference constraint rule would be used for processing. Or, proceed to the next step;

5. Calculate the fitness value of $P(g)$ and store the individual with the optimal $P(g)$ fitness in $B(g)$.

6. Add 1 to the evolutionary algebra, determine the quantum rotation angle $\theta$ according to (13), update the chromosome to obtain $Q(g+1)$, and simultaneously measure $V(g+1)$ to obtain the measured value $V(g+1)=\left\{v_{1}^{g+1}, v_{2}^{g+1}, \ldots, v_{s}^{g+1}\right\}$ then proceed to step (4) for processing;

7. Calculate the fitness value of $V(g+1)$ and store the individual with the max fitness among $V(g+1)$ into $B(g+1)$;

8. Determine whether the chromosome satisfied the variation threshold condition, and if it satisfied the variation threshold condition, mutate the chromosome; or go to the next step;

9. Determine if the maximum evolution algebra was reached, if so, terminate the procedure and output the final results; or, skip to step (6) for processing.

\section{IQGA Convergence Analysis}

To prove the convergence of IQGA, definitions and lemmas below were introduced [26], [27].

Definition 1. According to the definition of literature [27], set $\xi_{n}(n=1,2, \ldots)$ to be the random sequence defined in probability space. If there is a random variable $\xi$, set $p\left\{\lim _{n \rightarrow \infty} \xi_{n}=\xi\right\}=1$. Or if $\forall \varepsilon>0$, $p\left\{\bigcap_{n=1}^{\infty} \bigcup_{k \geq n}\left[\left|\xi_{k}-\xi\right| \geq \varepsilon\right]\right\}=0$, the random sequence $\xi_{n}(n=1,2, \ldots)$ would be converged to random variable $\xi$ with probability as 1 . Obviously, the convergence with probability 1 was stronger than convergence per probability. 
Definition 2. According to the definition of literature [26], set a random variable $f\left(q_{g}\right)=\max _{q_{i}^{g} \in Q_{g}}\left\{f\left(q_{g}^{t}\right) \mid\right.$ $i=1,2, \ldots, s\}=1$, which refers to the best fitness in the gth population status $Q_{g} . f^{*}$ refers to the globally optimal solution. Then the algorithm was converged to the globally optimal solution, only when $\lim _{g \rightarrow \infty} P\left\{f\left(q_{g}\right)=f^{*}\right\}=1$.

Lemma 1. According to literature [26], IQGA's chromosome sequence $\left\{Q_{g}, g \geq 0\right\}$ is a finite homogeneous Markov chain.

Lemma 2. Borel-Cantelli $(B-C)$, set $\varepsilon_{1}, \varepsilon_{2}, \ldots, \varepsilon_{n}$ as the independent event sequence in probability space, and $p\left(\varepsilon_{n}\right)$ is the probability. Then we get the following conclusion [27]:

$$
\begin{aligned}
& \sum_{n=1}^{\infty} p\left(\varepsilon_{n}\right)<\infty, \quad \text { then } \sum_{n=1}^{\infty} p\left(\bigcap_{n=1}^{\infty} \bigcup_{k=n}^{\infty} \varepsilon_{k}\right)=0 \\
& \sum_{n=1}^{\infty} p\left(\varepsilon_{n}\right)=\infty, \quad \text { then } \sum_{n=1}^{\infty} p\left(\bigcap_{n=1}^{\infty} \bigcup_{k=n}^{\infty} \varepsilon_{k}\right)=1
\end{aligned}
$$

According to the above two definitions and two lemmas, here it is to prove that after update and variation of dynamic adjustment of quantum rotation angle operation, the retained optimal individual IQGA may be converged to the globally optimal solution with probability 1 .

Demonstration: Set $Q_{g}=\left\{q_{1}^{g}, q_{2}^{g}, \ldots, q_{s}^{g}\right\}$ to be the population of generation $g$ in IQGA, where $s$ refers to population size, $q_{i}^{g}$ is the No. I quantum chromosome, and $v$ means the decimals. Then the state space size of $Q_{g}$ is $v^{n s} \subset R^{n}$. Set the feasible region to be calculated $\Omega=[a, b]=\left\{Q_{g} \subseteq v^{n s} \mid a_{i}<Q_{g}<b_{i}, \quad i=1,2, \ldots, s\right\}$ to be the bounded closed set, and $f(Q)$ is bounded continuous in $\Omega$, so there is an optimal solution $Q^{*}$ in $f(Q)$. As to $\forall Q^{*} \subseteq \Omega=\{Q \mid f(Q)=\max f(Q)\}$, when $\exists \varepsilon>0, Q \subseteq \Omega \cap\left\{\bar{Q}|| Q-Q^{*} \mid<\varepsilon\right\},\left|f(Q)-f\left(Q^{*}\right)\right|<\varepsilon$; set $D_{0}=\left\{Q \subseteq \Omega|| f(Q)-f^{*} \mid<\varepsilon\right\}, D_{1}=\Omega / D_{0}, I\left(Q^{*}\right)=$ $\left\{Q \subseteq v^{n s}|| Q-Q^{*} \mid<\varepsilon\right\}$, then $I\left(Q^{*}\right) \cap \Omega \subseteq D_{0}$; set $p_{i j}$ to be the probability when $Q_{g}$ is in status $a_{i} \in v^{\text {ns }}$ and $Q_{g+1}$ is in status $a_{j} \in v^{n s}$. When $i=1, j=0, Q_{g}$ is in status $a_{1}$, then the probability is $p_{10}$ when $Q_{g+1}$ is in status $a_{0}$. When $i=1, j=1, Q_{g}$ is in status $a_{1}$, then the probability is $p_{11}$ when $Q_{g+1}$ is in status $a_{1}$.

As $q_{i}^{g}$ is any individual in $Q_{g}$, after update and variation of dynamic quantum rotation angle, the individual is got to be $q_{j}^{g}$. $I\left(Q^{*}\right) \cap \Omega$ is the non-nullbounded closed area, and Lebesgue measure is $>0$, then $0<p\left(q_{i}^{g}\right)<1$. While $p\left(q_{j}^{g}\right)$ was continuous in feasible region $\Omega$ and it is a bounded closed set, then $\exists l \subseteq \Omega$. Thus $p(l)=\max \left\{p\left(q_{i}^{g}\right) \mid q_{i}^{g} \in \Omega\right\}$, and $0<p(l)<1$, therefore $p(l) \leq p\left(q_{j}^{g}\right)$. This proved that after update and variance of dynamic quantum rotation angle, the best individual was retained, allowing the population constantly to evolve along the optimum direction.

As the generation $g+1$ population relied on the population of generation $g$ in terms of conditional probability, the best individual in generation $Q_{g+1}$ population was better than that in $Q_{g}$. Thus $p_{10}=1$, and $p(l) \leq p\left(q_{j}^{g}\right) \leq p_{10}$. From the characteristics of probability amplitude of quantum bit, $p_{10}+p_{11}=1$, then $p_{11}=1-p_{10} \leq 1-p(l)$. If $c=1-p(l)<1, p_{11} \leq c<1$.

As to $\forall \varepsilon>0, p_{g}=p\left\{\left|f\left(Q_{g}^{*}\right)\right| \geq \varepsilon\right\}, f\left(Q_{g}^{*}\right)$ was the local best individual in generation $g$ population $Q_{g}$, then $p_{g}=\left\{\begin{array}{ll}0 & \exists k \in\{0,1, \ldots, g\}, Q_{g}^{*}(k) \in D_{0} \\ p_{g}^{*} & \forall h \in\{0,1, \ldots, g\}, Q_{g}^{*}(h) \in D_{1}\end{array}\right.$. In it $p_{g}^{*}=p\left\{Q_{g}^{*}(h) \in D_{1} \mid h \in\{0,1, \ldots, g\}\right\} . \quad$ So $p_{g}^{*}=p_{11}^{g} \leq c^{g}$, therefore $\sum_{g=0}^{\infty} p_{g}^{*} \leq \sum_{g=0}^{\infty} c^{g}$. Conclusively, the power series convergence theorem revealed that $\sum_{g=0}^{\infty} c^{g}$ was converged to $c /(1-c)$, so $\lim _{g \rightarrow \infty} \sum_{g=0}^{\infty} p_{g}^{*} \leq \lim _{g \rightarrow \infty} \sum_{g=0}^{\infty}$ $c^{g}=(c /(1-c))<0$.

The following can be inferred with $B-C$ lemma: $p\left\{\bigcap_{g=1}^{\infty} \bigcup_{h \geq g}\left[\left|f\left\{Q_{g}^{*}(h)\right\}-f^{*}\right| \geq \varepsilon\right]\right\}=0$.

Then it can be drawn with definition 1 : $p\left\{\lim _{g \rightarrow \infty} f\left\{Q_{g}^{*}(h)\right\}=f^{*}\right\}=1$, so the IQGA of the best individual retained was converged to the globally optimal solution with probability 1 after update and variance of dynamic adjustment of quantum rotation angle operation.

\section{Results and Analysis}

In this section, the feasibility and effectiveness of the IQGA will be first verified by selecting three continuous functions, then the spectrum allocation performance of the improved algorithm will be evaluated on account of the network average efficiency and time overhead.

\subsection{Test Functions and Result Analysis}

To verify the optimization ability and convergence speed, three typical complex continuous functions were selected and compared with the conventional QGA and GA. About 10 times of numerical optimization were performed with these three test functions to record the optimal value, average value, and average convergence algebra. Here the optimal value and the average value were used to measure the algorithm's optimization ability, while the average convergence algebra was used to measure the computational efficiency of the algorithm. The target functions are as follows:

$$
\left\{\begin{array}{l}
f_{1}\left(x_{1}, x_{2}\right)=x_{2} \cdot \sin \left(2 \pi x_{1}\right)+x_{1} \cdot \cos \left(2 \pi x_{2}\right)\left(-2 \leq x_{i} \leq 2, \quad i=1,2\right) \\
f_{2}\left(x_{1}, x_{2}\right)=21.5+x_{1} \cdot \sin \left(4 \pi x_{1}\right)+x_{2} \cdot \sin \left(20 \pi x_{2}\right)\left(-3 \leq x_{1} \leq 12.1,4.1 \leq x_{2} \leq 5.8\right) \\
f_{3}\left(x_{1}, x_{2}\right)=0.3 \cdot \cos \left(3 \pi x_{1}\right)+0.3 \cdot \cos \left(4 \pi x_{2}\right)-x^{2}-y^{2}-0.3\left(-10 \leq x_{i} \leq 10, \quad i=1,2\right)
\end{array}\right.
$$


Table 1

Data Comparison Table of Simulation

\begin{tabular}{|c|c|c|c|c|}
\hline & Method & Optimal Value & Mean of Value & $\begin{array}{c}\text { Average Convergence } \\
\text { Algebra }\end{array}$ \\
\hline$f_{1}$ & QGA & 3.7534 & 3.7121 & 137 \\
& GA & 3.5506 & 3.5127 & 143 \\
& Proposed method & 3.7563 & 3.7258 & 85 \\
\hline \multirow{2}{*}{$f_{2}$} & QGA & 38.8362 & 38.8176 & 139 \\
& GA & 38.6303 & 38.6243 & 158 \\
& Proposed method & 38.8503 & 38.8492 & 92 \\
\hline \multirow{2}{*}{$f_{3}$} & QGA & 0.2976 & 0.2965 & 162 \\
& GA & 0.2937 & 0.2924 & 169 \\
& Proposed method & 0.3 & 0.2995 & 112 \\
\hline
\end{tabular}

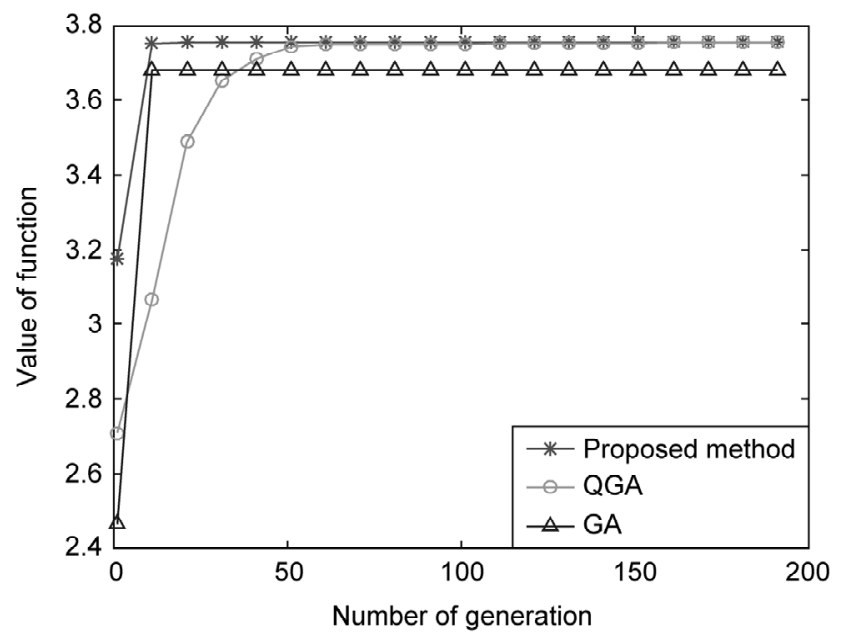

(a)

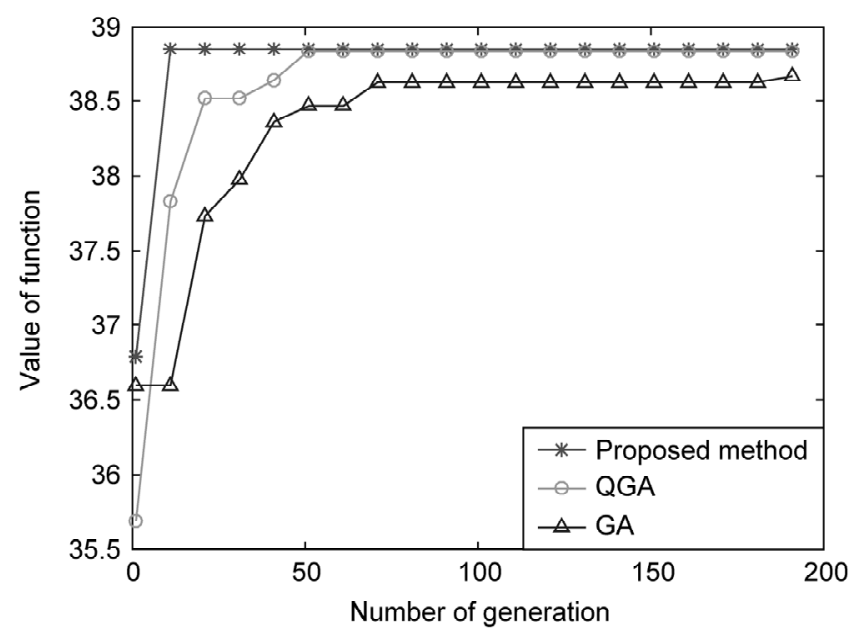

(b)

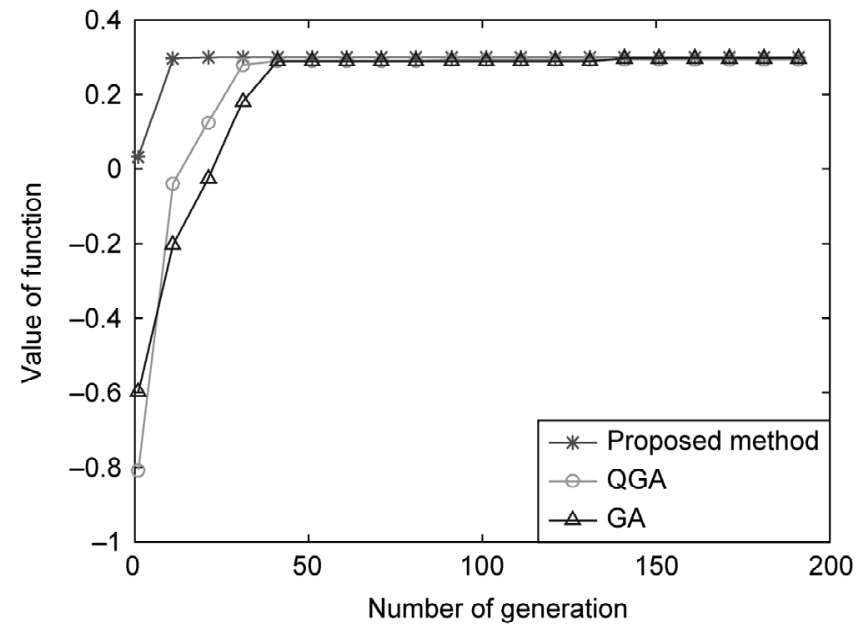

(c)

Figure 1. Comparison of $f_{1}-f_{3}$ optimal search: (a) Test function 1; (b) Test function 2; and (c) Test function 3.

Parameter settings of the algorithm in this work: when the population number was 30 and the chromosome length was 40 (the number of chromosomal genes in each variable was 20), the quantum rotation angle $\theta$ was determined by (13), and the mutation probability determined in Section 3.4; QGA parameter settings: when the population number was 30 and the chromosome length was 40 , the quantum rotation angle $\theta$ was $0.005 \pi$; parameter setting of GA algorithm: when the population number was 30 , by employing the binary string encoding method, the crossover probability was obtained as 0.9 and the mutation probability as 0.05 . The maximum evolution algebra of the three algorithms was 200. The data of the simulation is shown in Table 1, and the simulation curves are shown in Fig. 1(a)-(c). 
Table 1 shows that in terms of optimization ability, the optimal solution of the algorithm in this work was significantly higher than the other two algorithms. This result was accompanied by the average value of the searched optimal solutions that also reached the largest value, indicating that the proposed algorithm exhibited the highest stability. In terms of convergence speed, the mean convergence generations were also counted less than the numbers of other two algorithms, indicating a higher convergence rate. The simulation curves illustrate that the convergence speed of the proposed methodology could be more powerful. Looking at the simulation curves of Fig. 1(a)-(c), the convergence speed was much faster owing to the adaptive rotation angle, so it could quickly locate near the optimal solution in the early phase of the evolution algebra. This, undoubtedly, took the chance to make it easier to get the optimal solution. In summary, the proposed algorithm performed well enough in universal search capability with greater convergence speed than conventional methods.

\subsection{Spectrum Allocation Results and Analysis}

To verify the performance, the proposed algorithm was compared with the Color Sensitive Graph Coloring (CSGC), the Basic Quantum Genetic Algorithm (QGA), and the Genetic Algorithm (GA). At the same time, the indicators of network average efficiency and time overhead were also taken in to evaluate the spectrum allocation results of different algorithms. Specifically, the simulation results were introduced into the spectrum matrix $A$, utility matrix $B$, and interference constraint matrix $C$ in the experiment with reference to the pseudocodes provided in [28]. In the same experiment, all different algorithms used the same $A, B$, and $C$ matrices. The result of each experiment was average obtained by performing 40 algorithms separately. Parameter settings: the population number of all algorithms was 30, the evolution algebra 200, and the parameter design of CSGC referred to [9], with the rest to Section 5.1.

In the case where the number of primary users is $K=20$, frequency $b$ and $M=10$, and cognitive user $N=20$, the curve of the network average efficiency with the number of iterations is shown in Fig. 2. It can be seen from Fig. 2 that the proposed algorithm basically converges essentially at the 60th iteration and QGA converges at the 80th iteration. The difference is that converging action from other algorithms basically occurred only after the 120th iteration. During this process of verifying the convergence performance of the algorithm, the network average efficiency obtained by this algorithm was approximately $25 \%$ higher than other algorithms.

Figure 3 shows the change curve of the network average efficiency with the number of frequency bands $M$ in the case where the number of primary users is $K=20$, and the number of cognitive users is $N=20$. Please note that the average efficiency changed proportionately with the number of the frequency bands $M$, and higher than other algorithms. This further validated the effectiveness of this algorithm proposed for spectrum allocation.

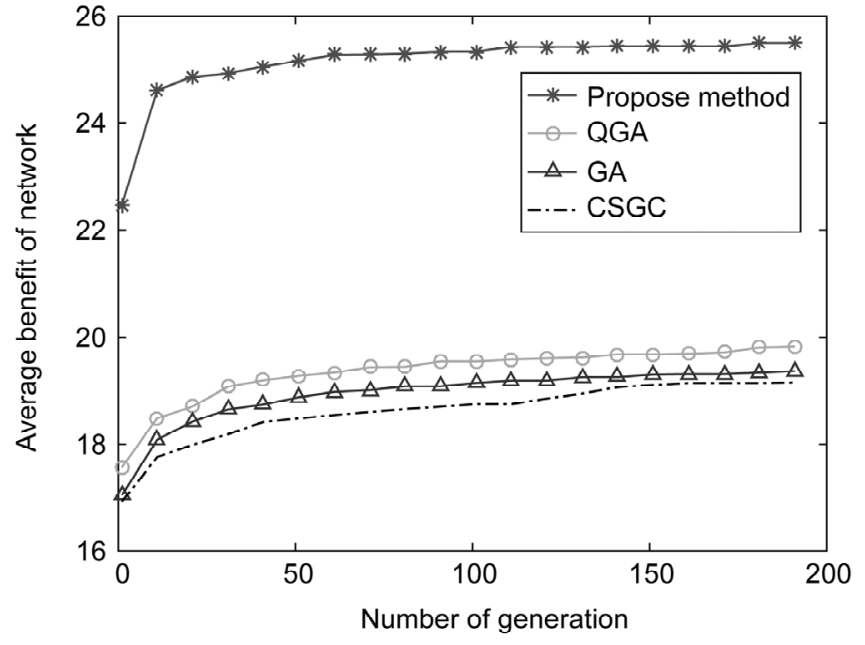

Figure 2. Comparison of network average benefit performance of different algorithms.

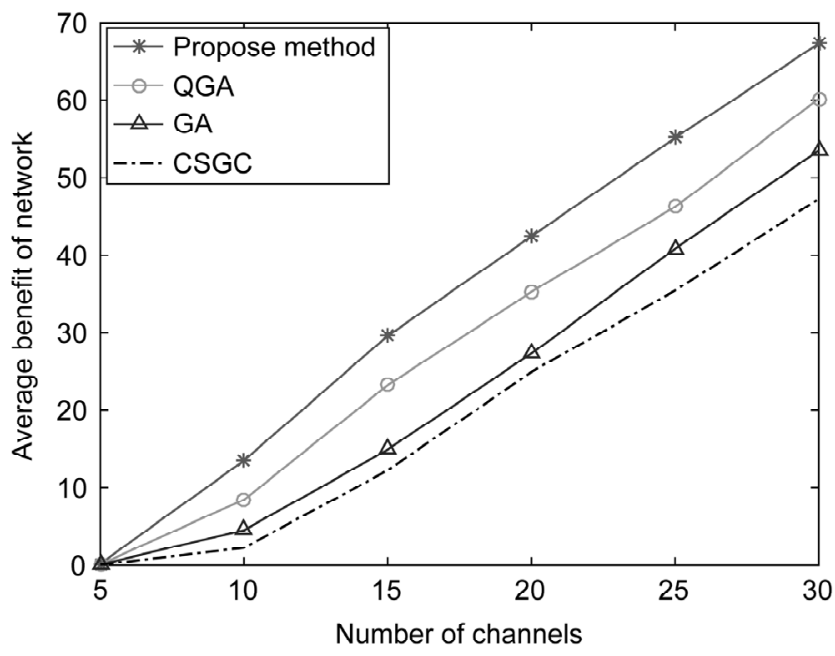

Figure 3. A graph of network average revenue and channel number of different algorithms.

Figure 4 shows the change curve of the network average efficiency with the number of cognitive users, where the number of primary users is $K=20$, and the number of frequency bands is $M=15$. As the number of cognitive users increases, the average efficiency shows a decreasing trend. The reason is as follows: as the cognitive users increased, the competition between cognitive users became more intense, and more interference occurred, so the average network efficiency decreased as the number of cognitive users increased. It can be seen from the simulation results that the performance of the proposed algorithm is significantly better than other algorithms, which is capable of enhancing the utilization of spectrum resources.

Figure 5 shows the change curve of the network average efficiency with the number of primary users, where the number of cognitive users is $N=20$, and the number of frequency bands is $M=10$. As the number of primary users increases, the average efficiency shows a decreasing trend. The reason is as follows: as the number of primary users increased, the number of available frequency bands decreased, and the cognitive users' competition for the 


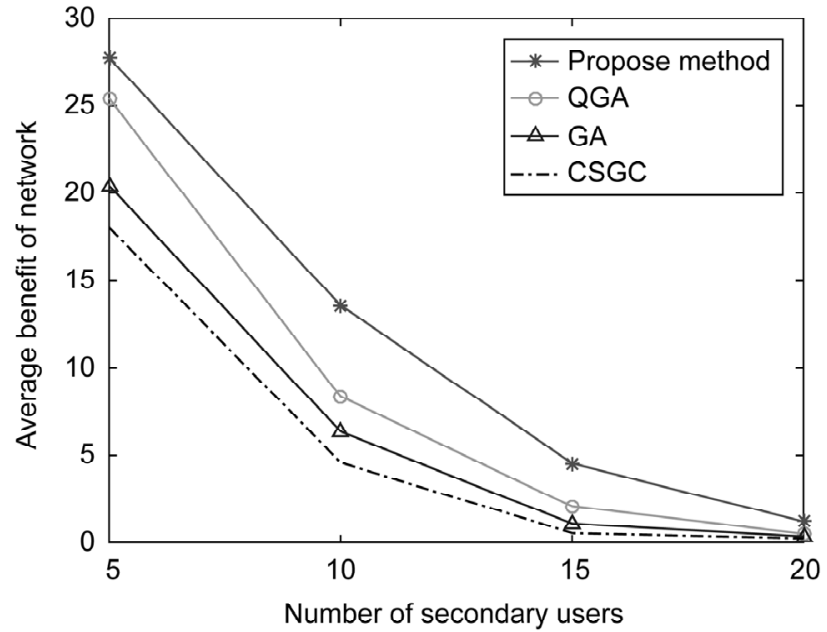

Figure 4. Graph of average network revenue and secondary users of different algorithms.

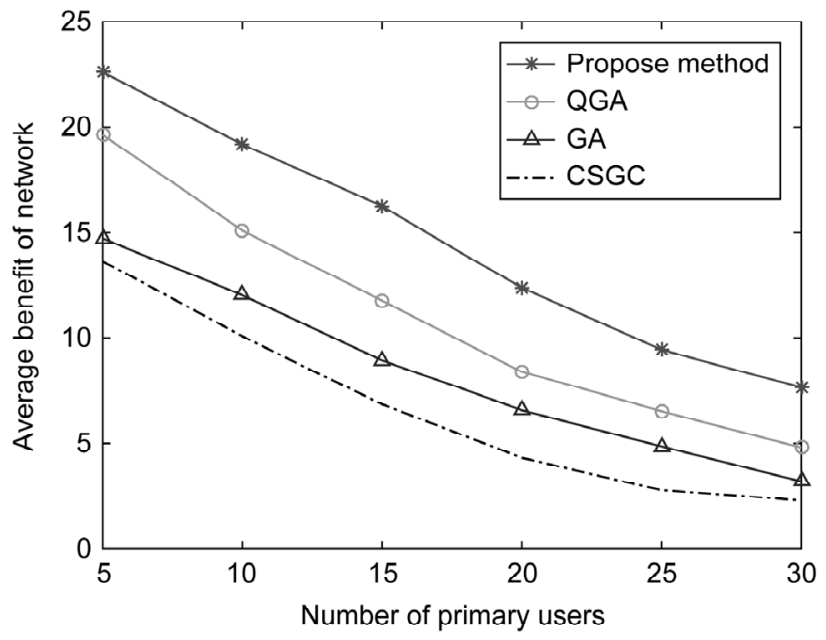

Figure 5. A graph of the average network revenue and the number of major users of different algorithms.

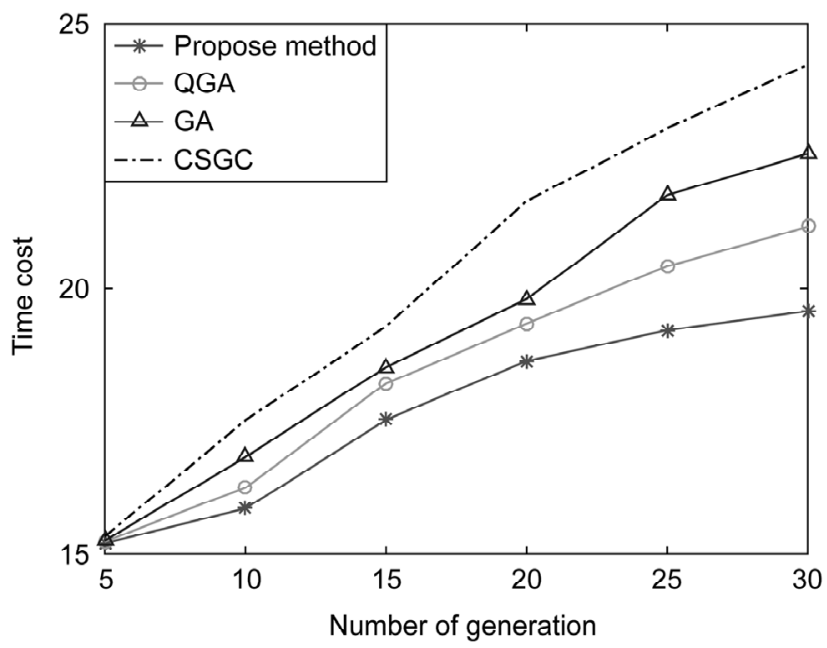

Figure 6 . The curve of time overhead as the number of iterations.

channel was increased. It can be seen from the simulation results that the performance of the proposed algorithm is obviously better than other algorithms, which can improve the utilization of spectrum resources.
Figure 6 shows a variation of the time overhead of the system with iteration times when the number of primary users is $K=20$, frequency bands is $M=5$, and cognitive users is $N=20$. It can be seen from Fig. 6 that as the number of users increased, the time overhead also increased, but the time overhead of the algorithm proposed in this paper is much smaller than other algorithms. The simulation results proved the efficiency of the algorithm.

\section{Conclusion}

Based on the analysis with the theoretical distribution model of graph colouring, a QGA for improving the energy efficiency of CR networks is proposed. First, the chaotic search algorithm was introduced into the chromosome initialization phase to enhance the diversity of the initial population. Second, the method of dynamically adjusting the quantum rotation angle accelerated the convergence speed of the algorithm, which effectively improved the operation efficiency of the algorithm. Finally, the mutation threshold was set to selectively mutate the chromosome to effectively solve the local optimal problem. At the same time, the reasonable interference constraint rules in the spectrum allocation process could further effectively solve the problem of channel ownership fairness. The simulation results show that by selecting three complex continuous functions for testing, the algorithm has better convergence speed and optimization ability. Moreover, network average efficiency and time overhead were applied to evaluate the spectrum allocation performance. Thus, it is evident that this algorithm can produce satisfied results with good convergence rate as to the enhancement of the network efficiency of the CRN system.

\section{Acknowledgement}

This work was mainly supported by the National Nature Science Foundation of China. (Grant No. 61379005).

\section{References}

[1] L. Li, C. Chen, Y. Wang, T. He, and X. Guan, Adaptive beacon transmission in cognitive-OFDM-based industrial wireless networks, IEEE Communications Letters, 21(1), 2017, $152-155$.

[2] E.U. Ogbodo, D. Dorrell, and A.M. Abu-Mahfouz, Cognitive radio based sensor network in smart grid: Architectures, applications and communication technologies, IEEE Access, 5(99), 2017, 19084-19098.

[3] B. Zhang, Y. Wu, X. Yi, and X. Yang, Joint communicationmotion planning in wireless-connected robotic networks: Overview and design guidelines, International Conf. on Communications (ICC), Kuala Lumpur, Malaysia, 2016.

[4] G. Stamatescu, D. Popescu, and R. Dobrescu, Framework for cognitive radio deployment in large scale WSN-UAV surveillance (Switzerland: Springer International Publishing, 2016), $41-56$.

[5] L. Deng, X. Ma, J. Gu, et al., Artificial immune network-based multi-robot formation path planning with obstacle avoidance, International Journal of Robotics and Automation, 31(3), 2016, 233-242.

[6] X.J. You, X.H. He, X.M. Han, et al., Cross-layer parameters reconfiguration in industrial cognitive wireless networks using MOABCHV algorithm, International Journal of Robotics and Automation, 33(2), 2018, 110-118. 
[7] J. Mitola, Cognitive radio: Software radios more personal, IEEE Personal Communications, 6(4), 1999, 13-18.

[8] G.F. Sun, X. Feng, X.H. Tian, et al., Coalitional double auction for spatial spectrum allocation in Cognitive Radio networks, IEEE Translations on Wireless, Communication, 13(6), 2014, 3196-3206.

[9] E. Driouch and W. Ajib, Downlink scheduling and resource allocation for cognitive radio MIMO networks, IEEE Translations on Vehicular Technology, 62(8), 2013, 3875-3885.

[10] H.L. Xiao, W.J. Zheng, Z.P. Nie, et al., Interference alignment for cognitive radio MIMO cognitive system based on game theory, Journal of University of Electronic Science and Technology of China, 46(5), 2017, 207-214.

[11] S. Sengupta and M. Chatterjee, Designing auction mechanisms for dynamic spectrum access, Mobile Networks and Applications, 13(5), 2008, 498-515.

[12] G. Na, S. Xiaoyan, G. Dunwei, et al., Solving robot path planning in an environment with terrains based on interval multi-objective PSO, International Journal of Robotics and Automation, 31(2), 2016, 100-110.

[13] J. Elhachmi and Z. Guennoun, Cognitive radio spectrum allocation using genetic algorithm, EURASIP Journal on Wireless Communications and Networking, 2016(1), 2016, 1-11.

[14] P. Lin and T.J. Yang, Spectrum allocation based on genetic particle swarm algorithm in cognitive radio system, Radio Engineering, 44(9), 2014, 12-15.

[15] Z.J. Zhao, Z. Peng, S.L. Zheng, et al., Cognitive radio spectrum assignment based on quantum genetic algorithm, Journal of Physics, 58(2), 2009, 1358-1363.

[16] C.C. Xiao, Researching on spectrum allocation in cognitive radio networks based on quantum genetic simulated annealing algorithm, China New Telecommunications, 17(24), 2015, 6-9.

[17] C. Shu, L.H. Sun, J.H. Li, and M.M. Gou, Improvement of quantum genetic algorithm and its application in cognitive radio spectrum distribution, Journal of Nano Electronics and Optoelectronics, 12, 2017, 1215-1218.

[18] Z. Zhao and Z. Peng, Cognitive radio spectrum allocation using evolutionary algorithms, IEEE Transactions on Wireless Communications, 8(9), 2009, 4421-4425.

[19] X.Y. Zhong, Channel allocation in cellular network based on self-adaptive genetic algorithm, Computer Engineering, 36(17), 2010, 189-191.

[20] Z.R. Wang, B. Yang, X.C. Lv, and D.W. Cui, Research on an improved quantum genetic algorithm, Journal of Xi'an University of Technology, 28(2), 2012, 145-151.

[21] G. Liu, H.Y. Zhao, H. Chen, et al., Spectrum allocation for cognitive radio network based on improved the quantum genetic algorithm, High Technology Communication, 25(8), 2015, 760-765.

[22] D.S. Fu and R. Zhang, Improved quantum genetic algorithm by balancing convergence and diversity, Journal of Computer Simulation, 30(12), 2013, 105-112.

[23] K.-H. Han and J.-H. Kim, On the analysis of the quantuminspired evolutionary algorithm with a single individual, IEEE Congress on Evolutionary Computation, Vancouver, Canada, 2006, 2622-2629.

[24] H.Y. Zhao, Spectrum allocation based on improved quantum genetic algorithm in cognitive radio network, Master Dissertation, Yanshan University, 2015.

[25] P. Lin and T.J. Yang, Spectrum allocation based on improved genetic algorithm in cognitive radio system, Computer Simulation, 31(2), 2014, 250-254.

[26] X. Zhang, R. Zhang, G. Sui, et al., Convergence analysis of double chains quantum genetic algorithm, Computer Engineering, 38(15), 2012, 127-132.

[27] W.X. Zhang and Y. Liang, Mathematical foundation of genetic algorithms (Xi'an, China: Xi'an Jiao Tong University Press, 2000).

[28] C.Y. Peng, H.T. Zhang, and B.Y. Zhao, Utilization and fairness in spectrum assignment for opportunistic spectrum access, Mobile Networks and Application, 11(4), 2006, 555-576.

\section{Biographies}

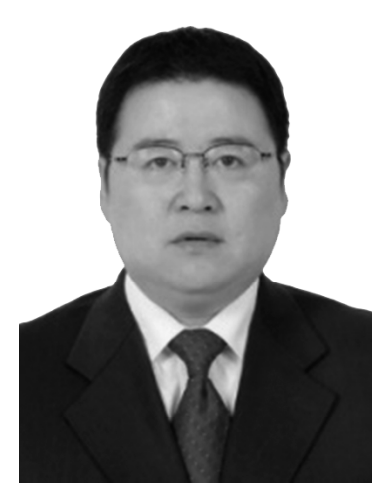

Bin Han received the B.E. and M.E. degrees from Southwest University of Science and Technology, Mianyang, China, in 1997 and 2009, respectively. He is currently a $\mathrm{Ph} . \mathrm{D}$. candidate in the Southwest University of Science and Technology. His research interests include wireless broadband communications and network resource management.

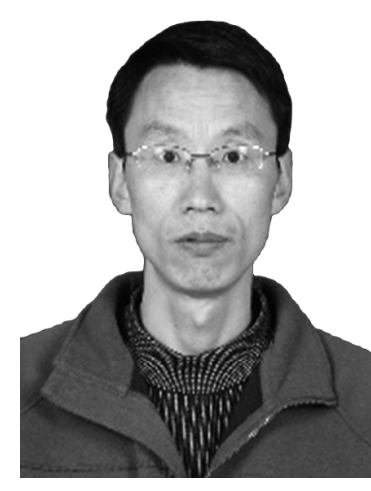

Hong Jiang received his Ph.D. degree in School of Communication and Information Engineering from University of Electronic Science and Technology of China. He is a full professor at the South West University of Science and Technology of China. His current interests include the cross layer QoS support in ad hoc networks and intelligent learning for resource management in cognitive

radio networks.

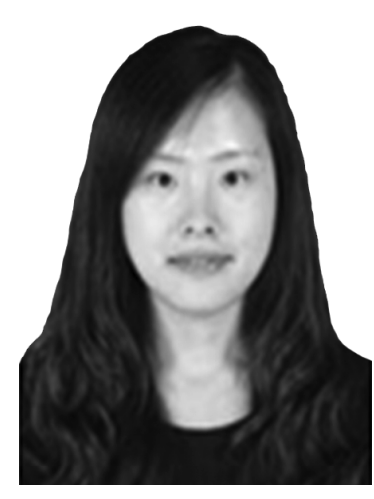

Ying Luo was graduated with the B.S. and M.S. degrees from the Department of Information Engineering, South West University of Science and Technology (SWUST), in 2012 and 2015, and received her Ph.D. degree from the Department of Electronic Engineering and Information Science (EEIS), University of Science and Technology of China (USTC), in 2018. Currently, she is a teacher in the Department of Information Engineering, SWUST. Her research interests include next-generation networks.

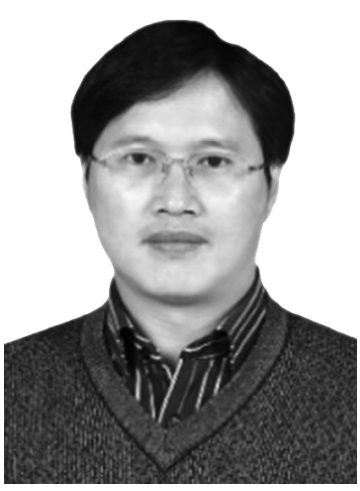

Jinzhi Zhou received the B.E. and M.E. degrees from Changchun Institute of Optics and Fine Mechanics and University of Electronic Science and Technology of China, in 1995 and 2002, respectively. He is currently a Ph.D. candidate in the Southwest University of Science and Technology. His research interests include computer network and Internet of Things and machine learning. 